\title{
Stratospheric QKD: Feasibility Analysis and Free-Space Optics System Concept
}

\author{
Florian Moll*a $^{\mathrm{a}}$, Thierry Botter ${ }^{\mathrm{b}}$, Christoph Marquardt ${ }^{\mathrm{c}}$, David Pusey ${ }^{\mathrm{b}}$, Amita Shrestha $^{\mathrm{a}}$, Andrew \\ Reeves $^{\mathrm{a}}$, Kevin Jaksch ${ }^{\mathrm{c}}$, Kevin Guenthner ${ }^{\mathrm{c}}$, Oemer Bayraktar ${ }^{\mathrm{c}}$, Christian Mueller-Hirschkorn ${ }^{\mathrm{c}}$, \\ Alberto Diago Gallardo ${ }^{\mathrm{d}}$, Dionisio Diaz Gonzalez ${ }^{\mathrm{d}}$, Wenjamin Rosenfeld ${ }^{\mathrm{e}}$, Peter Freiwang ${ }^{\mathrm{e}}$, Gerd \\ Leuchs $^{\mathrm{c}}$, Harald Weinfurter ${ }^{\mathrm{e}}$ \\ ${ }^{\mathrm{a}}$ German Aerospace Center, Oberpfaffenhofen, Muenchener Strasse 20, 82234 Wessling; ${ }^{\mathrm{b}}$ Airbus \\ Defence and Space GmbH, Willy-Messerschmitt-Strasse 1, 82024 Taufkirchen; ${ }^{\circ}$ Max Planck \\ Institute for the Science of Light, Staudtstr. 2, 91058 Erlangen; ${ }^{d}$ Mynaric Lasercom GmbH, \\ Dornierstr. 19, 82205 Gilching; ${ }^{\mathrm{e}}$ Ludwig-Maximilians-Universität Muenchen, Schellingstr. 4, 80799 \\ Muenchen
}

\begin{abstract}
Quantum key distribution (QKD) is one of the most mature quantum technologies and can provide quantum-safe security in future communication networks. Since QKD in fiber is limited to a range of few hundred kilometers, one approach to bridge continental scale distances may be the use of high altitude pseudo satellites (HAPS) as mobile trusted nodes in the stratosphere. In parallel, free-space laser communication for high rate data transmission has been a subject of research and development for several decades and its commercialization is progressing rapidly. Important synergies exist between classical free-space communication and QKD systems since the quantum states are often implemented using the same degrees of freedom such as polarization or field amplitude and phase. These synergies can be used to benefit from the progress in classical free-space laser communication in QKD applications.

In this paper, the use case of QKD in a stratospheric environment is described wherein HAPS may serve as relay station of secret keys and encrypted data. The mission scenario and HAPS capabilities are analyzed to derive special requirements on the stratospheric laser terminal, the link geometry and the ground segment with respect to a feasibility demonstration.

To obtain a flexible and compatible system, discrete variable and continuous variable QKD protocols are considered to be implemented side by side in the HAPS payload. Depending on the system parameters, it can be beneficial to use the one or the other kind of protocol. Thus, a direct comparison of both in one and the same system is of scientific interest. Each of the protocols has particular requirements on coupling efficiency and implementation.

Link budget calculations are performed to analyze possible distances, key rates and data transmission rates for the different schemes. In case of the QKD system, the mean coupling efficiency is of main interest, i.e. signal fluctuations arising from atmospheric turbulence must be taken into account in the security proof, but the buffered key generation relaxes real-time requirements. This is different to classical communications, where the corresponding fading loss must be assessed. A system architecture is presented that comprises the optical aircraft terminal, the optical ground terminal and the most important subsystems that enable implementation of the considered QKD protocols. The aircraft terminal is interfaced with the dedicated quantum transmitter module (Alice) and the ground station with the dedicated quantum receiver module (Bob). The optical interfaces are SMF couplings which put high requirements on the receiving optics, in particular the need for wave-front correction with adaptive optics. The findings of the system study are reviewed and necessary next steps pointed out.
\end{abstract}

Keywords: Quantum Key Distribution, Free-Space Optical Communications, stratospheric communications, communications system design

corresponding author: florian.moll@dlr.de 


\section{INTRODUCTION AND MOTIVATION}

\subsection{General}

Quantum Key Distribution enables two communicating parties to generate private keys whose security can be certified based on quantum mechanical principles [1], [2]. The topic has been an active field of scientific investigation for several decades and has recently matured to the point of producing first commercially viable solutions for terrestrial (fiberbased) communication, e.g. [3], [4]. The further development of the technology towards free-space applications is currently a major focus point of research, one with even national security implications. First airplane- and satellite-based demonstrations have been realized, though with limited relevance for industrial applications [5-9]. Additional spaceborne technology demonstrations, several with involvement of industrial players, have been announced and are currently in development, e.g. [10], [11].

This feasibility study examines the possibility of a technology development to realize QKD from a stratospheric platform and the ground. We analyze the implementation of a side-by-side exploitation of continuous variable (CV-) and discrete variable (DV-) QKD. This combination would benefit from the advantages of the two schemes, operation even at daylight and over long distances during night.

The example stratospheric platform is chosen to be the Zephyr developed by Airbus Defence and Space. The Zephyr-S is currently Airbus' only stratospheric platform in operation. It is the 8th model in the string of Zephyr stratospheric systems developed and closely resembles its immediate predecessor, the Zephyr-7. The two most recent Zephyrs differ only in small structural improvements.

\subsection{Use Case Stratospheric QKD: HAPS as relay stations for secret keys}

HAPS could relay QKD links over intermediate distances via a direct trusted relay link, i.e. by sharing keys to overhead satellites and / or to nearby ground stations, or via a trusted network of multiple neighboring HAPS. More generally, HAPS could enable ad hoc deployment of secret key distribution and optical communication around areas where this service is needed.

As a general remark, one should stress that the QKD key exchange is a buffered operation and does not need real-time communication of the secret messages. The QKD key exchange will fill up key stores with secret keys between ground stations / satellites and HAPS or between different HAPS in a relay situation. Those key stores can then be used to encrypt secret messages with any conventional encryption method, such as one-time-pad or AES, and transmit them by any means and at any time until the key store runs out of secret keys. The size of the key stores should be carefully chosen depending on the user requirements and available QKD key rate. The key store should be large enough to take into account the unavailability of the QKD link, e.g. due to adverse weather conditions.

\section{CHANNEL MODELLING}

\subsection{Link Geometry}

The geometry of the link depends on various parameters, such as the link distance, elevation angle and angular speeds reached by the flight terminal (FT). There are several limitations to the ground station, which can limit the constellation of the ground station location and the flight trajectory. One of these limitations is a singularity caused by the mounting of the telescope. Coming close to $90^{\circ}$ elevation, the telescope has a tracking speed limit which limits the speed an object can be tracked. This affects mainly low Earth orbit to ground links or aircraft ground links. A rather conservative maximum elevation angle of $75^{\circ}$ is therefore used to avoid this issue. However, this angle can certainly be increased considering careful tuning of the motor and motor control. Another limitation is the possibility of cables winding themselves around the telescope base, as the ground station tracks the stratospheric platform circulating directly overhead. This situation can be avoided by placing the ground station outside the aircraft's circular trajectory. The HAPS would corkscrew up and down in the diurnal cycle while communicating with the Transportable Optical Ground Station (TOGS) [12]. This trajectory describes a cylinder which tangential points towards the TOGS define the link distance and viewing angle.

\subsection{The Free-Space Channel}

The channel model focusses on description of the mean end-to-end loss between Alice and Bob. It can also be considered as the mean coupling efficiency between the partners. The relevant phenomena are outlined below. 
Free-space propagation: the transmit spatial mode is modelled as a Gaussian beam with beam quality factor M2. Gaussian beam propagation theory is then used to determine the sole signal loss due to the beam footprint overcasting the receiving telescope.

Atmospheric extinction: atmospheric extinction comprises scattering and absorption due to atmospheric molecules and aerosols. The molecular absorption defines the useable windows in the atmospheric transmission spectrum. Scattering on molecules (small particles) is described by Rayleigh scattering and scattering on aerosols (large particles) by Mie scattering. The composition of the gases and meteorological parameters in the current example is defined by the mid latitude summer atmosphere model in combination with the desert aerosol model. The HITRAN database is used to define the extinction properties of the gases for the given atmosphere model.

Atmospheric turbulence: as the beam propagates it experiences wave-front distortions leading to constructive and destructive interference across the beam. This causes different effects like intensity scintillation, angel-of-arrival fluctuations, beam wander, beam broadening and wave-front distortion as is. In the actual scenario, only wave-front distortion is regarded. Other effects are considered to be marginally affecting.

\subsection{Link budget considerations}

Link budgets were calculated for the DV and CV QKD in downlink, and free-space optical (FSO) communications in downlink and uplink direction for $30 \mathrm{~km}$ link distance each. The system design is based on the selection of existing airborne and ground laser terminals that can be extended with QKD systems. Therefore, the systems are not necessarily fully optimized and room for improvement exists. Following assumptions and simplifications are taken: Clear aperture diameter of the Micro Laser Terminal, MLT, (i.e. optical communications payload) is $56 \mathrm{~mm}$, Rx aperture of the TOGS is $600 \mathrm{~mm}$, Tx beam has Gaussian profile, pointing penalty in downlink is $3 \mathrm{~dB}$ (assuming appropriate performance for $850 \mathrm{~nm}$ and $1550 \mathrm{~nm}$ ), Beam wander is negligible, Fried parameter is $8 \mathrm{~cm} @ 850 \mathrm{~nm}$, atmospheric wind speed is $25 \mathrm{~ms}$ , coupling loss is $4 \mathrm{~dB} @ 1560 \mathrm{~nm}$ and $8 \mathrm{~dB} @ 850 \mathrm{~nm}$ (limited by the reflectivity of the aluminum-coated mirror and the performance of the adaptive optics system), and atmospheric transmission of $1.5 \mathrm{~dB} @ 1550 \mathrm{~nm}$ and $1.7 \mathrm{~dB} @ 850$ $\mathrm{nm}$. We consider that only mean losses are relevant for the quantum channel. The resulting losses for the DV and CV QKD downlink are $26 \mathrm{~dB}$ and $22 \mathrm{~dB}$, respectively. In the case of classical communication, the link margin with respect to a given data receiver sensitivity and transmit power is of interest. Here, the link margin for the $1 \mathrm{Gbps}$ link is $17 \mathrm{~dB}$ for the downlink signal (assumed Rx-sensitivity $-39.4 \mathrm{dBm}$ and $100 \mathrm{~mW}$ Tx-power) and $2.2 \mathrm{~dB}$ for the uplink signal (assumed Rx-sensitivity $-39.4 \mathrm{dBm}$ and $2 \mathrm{~W}$ Tx-power).

\section{SYSTEM CONCEPT}

\subsection{Architecture}

The overall block diagram of the HAPS-QKD system is shown in Figure 1. Green blocks show the aircraft and ground support equipment, default experiment system of the HAPS. The QKD sender and transmitter devices (CV and DV QKD) are marked as purple blocks. The red blocks show the airborne laser terminal and the belonging ground control equipment. The downlink laser beams are colored appropriately. The black and white telescope sketch with bright blue print is the TOGS. The beacon uplink is indicated with a bright blue arrow. The free-space beam is received by the telescope and coupled to a single mode fiber using an integrated adaptive optics system. 
Figure 1: Block diagram of the z-QKD system architecture. ANT: antenna; GCS: Ground Control Segment; MLT: Micro Laser Terminal; TOGS: Transportable Optical Ground Station; QKD: Quantum Key Distribution; UHF: Ultra High Frequency.

\subsection{Suitable QKD protocols}

QKD provides unprecedented security as it enables the analysis of the maximum information an eavesdropper can obtain from the communication channel. For this purpose, encoding in nonorthogonal quantum states of light is used together with classical authenticated communication for deducing the secret key from the detection events. Various schemes have been designed for encoding and key generation, which mainly differ in the hardware requirements and performance under different conditions. Here, we describe the most common and best studied protocols to enable decision on the protocol to be used.

The first protocol, proposed by Bennett and Brassard in 1984 (BB84) [1], [13], uses four quantum states, which are pairwise mutually orthogonal, thereby forming two bases. The two bases are oriented such that the eigenstates of one basis are maximally uncertain in the other basis. For the polarization of light typical orientations are vertical (encoding bit value " 1 "), horizontal ("0"), $+45^{\circ}$ (" 1 ") and $-45^{\circ}$ ("0") linear polarization. Another possibility, typically employed in fiber-based QKD is using the phase difference between two coherent pulses. Possible phase differences for this type are $0, \pi / 2, \pi, 3 \pi / 2$.

The protocol relies on the fact that when using single quanta of light, the eavesdropper can make maximally a single measurement on the sent signal. Yet, as the generation of single photons requires dedicated, complex hardware, variations of the protocol have been developed, which also allow the use of attenuated pulses of light. The general problem for this case is the occurrence of pulses with more than one photon. Together with the limited transmission, the so-called photon number splitting (PNS) [14] attack allows an eavesdropper to extract information about the states. Socalled decoy-state protocols [15] use additional pulses with different attenuation to detect possible attacks on the photon number. As they enable to again determine the maximum information an eavesdropper might have, security analysis and key sifting protocol is equal to the case for BB84 with single photons and results, up to some factor, in a similar dependence of the secure key rate.

The generic scheme of the BB84 protocol can be varied in several ways to optimize for distance and especially for the rate of sent signals. Changing the encoding sequence, e.g., to $\mathrm{H} / \mathrm{V}$ polarization encoding " 0 " and diagonal polarization encoding "1", the SARG04 [16] protocol has a higher persistence against the PNS attack, or by directly concatenating the pulse pairs of phase-encoded signals the distributed phase shift coding [17] and the COW [18] protocol reach the maximum rate for given pulse durations.

In contrast to DV QKD, where single photon detection is used, continuous variable QKD uses the technique of homodyne detection to measure the quadrature components of the electric field, similar to classical coherent communication. In the proposed mission, we plan to use a Gaussian modulated coherent state protocol, as already used in various experiments [19-21]. Alice prepares coherent states which amplitudes in phase space are chosen according to 
a Gaussian probability distribution. Bob then measures these coherent states either by homodyne or heterodyne detection, i.e., measuring one or two quadrature components at the same time. After the transmission of the quantum states is finished and a raw key is established, Alice and Bob have to conduct several classical post-processing steps in order to create the final secret key [22], [23]: first, they publicly compare a subset of the data to estimate the channel transmission and the excess noise of the system. Here, fluctuating channel parameters have to be taken into account by monitoring the transmission and effectively binning into sub-channels [24]. Afterwards, they perform an error correction procedure to create a common key out of their noisy data. In a last step, the information of a potential eavesdropper is reduced by applying a family of universal hash functions to the data, leading to the final secret key.

\subsection{QKD Payloads}

The QKD payload contains two detached modules for CV and DV QKD, respectively. The DV QKD module could employ four laser sources (vertical cavity surface emitting lasers, VCSELs) for generation of faint pulses with 4 different polarizations of the BB84 protocol. The four lasers are arranged in a linear array with $250 \mu \mathrm{m}$ pitch. The polarization of the emitted light is defined by four micro structured wire-grid polarizers and coupled into a small waveguide circuit for combination into a single spatial mode. The output of the circuit is then coupled into a single-mode fiber for connection with the optical terminal. Typical performance parameters with VCSEL diodes operating at $805 \mathrm{~nm}$ would be a repetition rate of 100-300 MHz and pulse duration of about $200 \mathrm{ps}$. Given a high timing resolution at the detectors and receiver electronics, this enable the important suppression of detector dark counts and background light. Using micro-optics the whole module can be highly integrated and requires a size of only $3 \times 3 \times 30 \mathrm{~mm}^{3}$.

The CV QKD module is a chip-based system using indium phosphide technology, which is developed as part of the $\mathrm{BMBF}$ project QUBE. It is designed to generate strongly attenuated laser pulses that can be modulated in amplitude and phase.

In order to compensate for the temperature variations that can occur during the mission, both QKD modules will be temperature stabilized. A payload computer will control the QKD modules and also serves as the post-processing unit for the QKD protocols.

\subsection{Airborne terminal}

In order to transmit the classic channel and the QKD signals on the airborne platform, the MLT-70 from Mynaric is chosen. The decision was made based on the experience of Mynaric on airborne laser communication payloads and the previously demonstrated capabilities of the MLT-70 [25]. The system is designed for stratospheric applications offering competitive Size, Weight and Power (SWaP) properties as well as a high temperature range. The current design of the system can be seen in Figure 2.

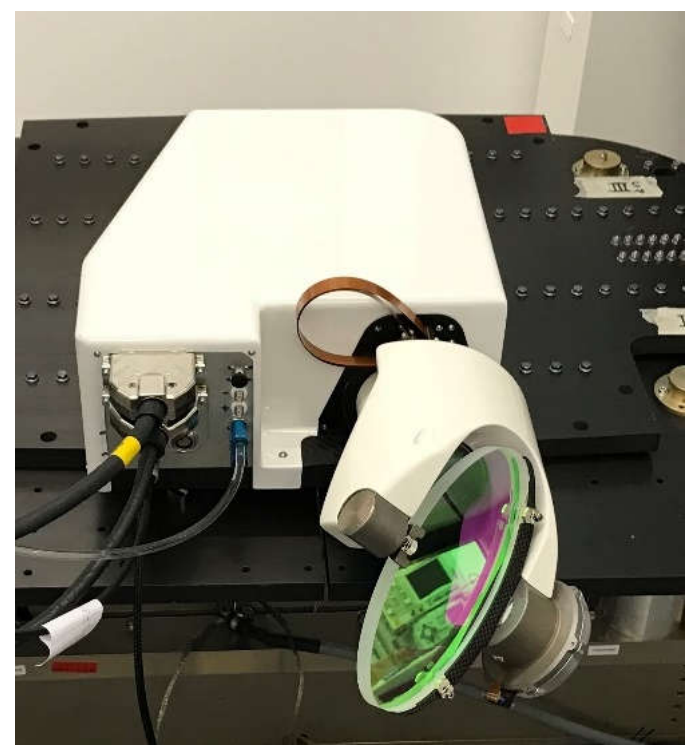

Figure 2: System view of the current MLT-70. 
The MLT-70 consists of two different modules, the electronics box and the optical module.

Electronics box: This unit includes all electronic components which control the MLT-70 as well as the different peripherals inside: the electronics power supply, the control computer, the control of sensors and the control for the EDFA module. Included in this box is also the laser transceiver. The interfaces to this box include optical fibers for the uplink and downlink wavelengths, the Ethernet connection for data transfer, the connection to the power supply, an Ethernet connection to send commands and receive status messages from the MLT-70 and the SMA connection used for the clock synchronization channel, as well as electrical harnesses to power and control peripherals in the optical module. The separation between two modules presents the benefit that the electronics box can be placed in the wing tips in order to save weight in the nose payload bay. There is also an option to split the electronics box into two units, although this option would involve major changes in the power distribution design of the MLT-70 and should therefore just be used as a backup.

The optical module: This unit includes all optical elements of the system. This includes all lenses and optical elements to differentiate between uplink and downlink, as well as all sensors, i.e. the receiver front-end for data reception (RFE) and the tracking sensors. Included in this module are the transmitter collimators, which feature a 50- $\mu$ rad collimator onaxis to be used by the QKD channel and a 400- $\mu$ rad transmitter for both tracking and classical FSO channel communications. The interfaces to this module include: the counter connection to the electrical harness from the electronics box, the counter fibers (fibers from the electronics box to the transmitter and from the receiver to the electronics box), the input fibers for $1550 \mathrm{~nm}$ and $850 \mathrm{~nm}$ from the QKD payload and the free-space input and output signals from the $70 \mathrm{~mm}$ aperture of the optical head.

Figure 3 shows the resulting block diagram from the airborne QKD payload together with the interfaces to the MLT-70. The wavelength of the CV-QKD module was set to $1560 \mathrm{~nm}$. The optical up- and downlinks for the classical channels were chosen to be at $1590 \mathrm{~nm}$ and $1545 \mathrm{~nm}$, respectively. This bidirectional optical link serves as public classical channel required by the QKD system for reconciliation, error correction and privacy amplification. A bidirectional Ethernet connection between the QKD payload computer and the MLT transceiver is used to exchange the classical information with the optical terminal. The QKD module needs to be time synchronized with the MLT-70 in order to assign and process the QKD data and the classical data. Especially the DV QKD demands highly precise synchronization ( 200 ps timing jitter), which can be achieved via a dedicated master clock connection between the MLT-70 transceiver and the QKD module.

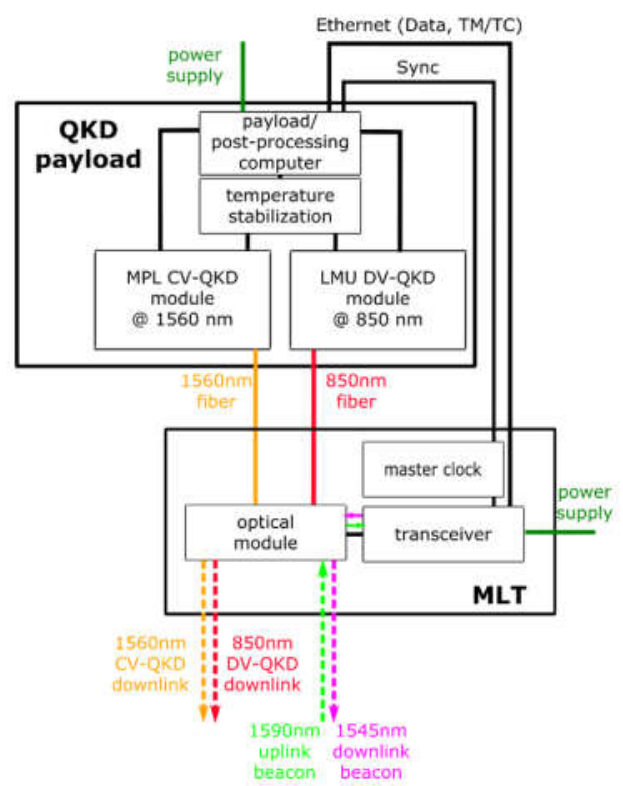

Figure 3: Block diagram for interfaces between QKD payload and MLT-70. TM/TC: TeleMetry/TeleControl; MLT: Micro Laser Terminal. 


\subsection{Ground station}

The concept of the ground segment is shown in Figure 4. FSO (Free-Space Optics) denotes all optical up- and downlinks. The blue lines denote high frequency electrical connections (public channel and synchronization markers), the red lines, optical fiber connections, and the green lines, data connections, i.e. public channel and TMTC (TeleMetry $\&$ TeleControl).

Z-QKD GND Block Diagram

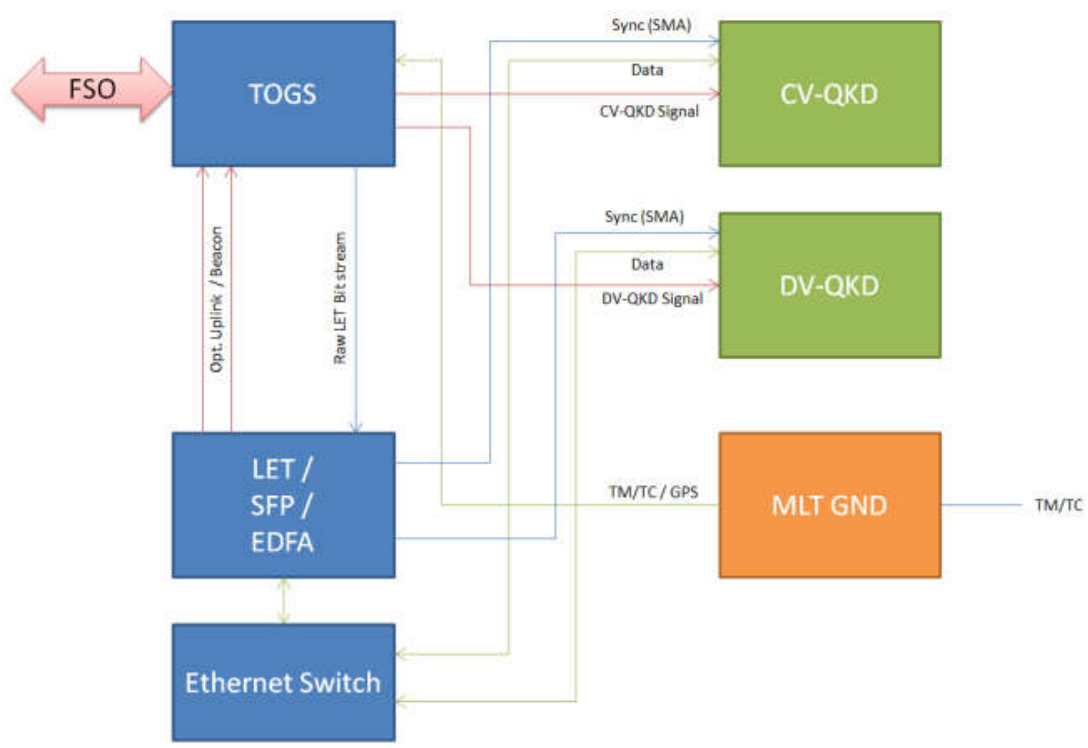

Figure 4: Concept of ground segment. FSO: Free Space Optics; TOGS: Transportable Optical Ground Station; LET: Laser Ethernet Transceiver; SFP: Small Form factor Pluggable; EDFA: Erbium Doped Fiber Amplifier; MLT GND: Micro Laser Terminal Ground Station

In the current state the optical path behind the main telescope is split into two beams by a 90:10 beam splitter cube. On the upper path, which has a splitting ratio of $90 \%$ the receiver front-end is located. On the straight path with a split ratio of $10 \%$ the acquisition and tracking camera is mounted. To interface the QKD receivers systems with single mode fibers, integration of an adaptive optics system to correct for wave-front aberrations is necessary. Location of the AO compartment in the TOGS is shown in Figure 5, left. The straight path is still used for the acquisition and tracking camera. The reflection path, originally hosting the RFE contains now the AO system with fiber coupling. Thus, the QKD receivers can be built as discrete system blocks standing aside the TOGS telescope. 


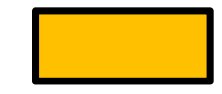

Figure 5: CAD cut of TOGS telescope and electronics box (left) and image of TOGS with unfolded telescope (right).

\section{DISCUSSION AND OUTLOOK}

The focus of the current work is to figure out the feasibility of integrating QKD systems to specific existing laser communications platforms. The system concept shows that it is possible and feasible. The link budget calculation shows that the overall coupling efficiency necessary for achieving a useful key rate is met. The key rates that can be obtained are in the order of several kbps with $100 \mathrm{MHz}$ transmit pulse repetition rate. It must be noted here, that the calculated coupling efficiencies are not for a fully optimized system but for specific platforms with some non-ideal optical elements, e.g. the mirror coatings of the TOGS. Nevertheless, it shows that the QKD system can run with appropriate performance. In conclusion, the QKD systems can be integrated in a stratospheric platform and typical laser terminals with moderate effort.

The next recommended steps of the work comprise improvement of the coupling efficiency to obtain still higher key rates, investigation of optimal background noise suppression methods and analysis of the environmental impact on the individual subsystems.

\section{ACKOWLEDGEMENTS}

We acknowledge the contribution of Mr. Martin Brechtelsbauer who contributed much to the system design and the ground station design.

\section{REFERENCES}

[1] C. H. Bennett and G. Brassard, "Quantum cryptography: Public key distribution and coin tossing," in Theor. Comput. Sci. 560, 1984.

[2] C. H. Bennett, "Quantum cryptography using any two nonorthogonal states," Phys. Rev. Lett., vol. 68, no. 21, pp. 3121-3124, May 1992.

[3] "ID Quantique." 2019.

[4] "QuantumCTek." 2019.

[5] S. Nauerth, F. Moll, M. Rau, C. Fuchs, J. Horwath, S. Frick, and H. Weinfurter, "Air-to-ground quantum communication," Nature Photonics, vol. 7, pp. 382-386, 2013.

[6] S.-K. Liao, W.-Q. Cai, J. Handsteiner, B. Liu, J. Yin, L. Zhang, D. Rauch, M. Fink, J.-G. Ren, W.-Y. Liu, Y. Li, Q. Shen, Y. Cao, F.-Z. Li, J.-F. Wang, Y.-M. Huang, L. Deng, T. Xi, L. Ma, T. Hu, L. Li, N.-L. Liu, F. Koidl, P. Wang, Y.-A. Chen, X.-B. Wang, M. Steindorfer, G. Kirchner, C.-Y. Lu, R. Shu, R. Ursin, T. Scheidl, C.-Z. Peng, J.-Y. Wang, A. Zeilinger, and J.-W. Pan, "Satellite-Relayed Intercontinental Quantum Network," Phys. Rev. Lett., vol. 120, no. 3, p. 030501, Jan. 2018. 
[7] C. J. Pugh, S. Kaiser, J.-P. Bourgoin, J. Jin, N. Sultana, S. Agne, E. Anisimova, V. Makarov, E. Choi, B. L. Higgins, and T. Jennewein, "Airborne demonstration of a quantum key distribution receiver payload," Quantum Science and Technology, vol. 2, no. 2, p. 024009, Jun. 2017.

[8] S.-K. Liao, W.-Q. Cai, W.-Y. Liu, L. Zhang, Y. Li, J.-G. Ren, J. Yin, Q. Shen, Y. Cao, Z.-P. Li, and et al., "Satellite-to-ground quantum key distribution," Nature, vol. 549, no. 7670, pp. 43-47, Aug. 2017.

[9] K. Günthner, I. Khan, D. Elser, B. Stiller, Ö. Bayraktar, C. R. Müller, K. Saucke, D. Tröndle, F. Heine, S. Seel, P. Greulich, H. Zech, B. Gütlich, S. Philipp-May, C. Marquardt, and G. Leuchs, "Quantum-limited measurements of optical signals from a geostationary satellite,” Optica, vol. 4, no. 6, pp. 611-616, Jun. 2017.

[10] R. Haber, D. Garbe, K. Schilling, and W. Rosenfeld, "QUBE - A CubeSat for Quantum Key Distribution Experiments," in Proceedings of 32nd Annual AIAA/USU Conference on Small Satellites, 2018.

[11] "SES Announces 10 Project Partners in QUARTZ Satellite Cybersecurity Consortium.” 2019.

[12] F. Moll, A. Shrestha, and C. Fuchs, "Ground stations for aeronautical and space laser communications at German Aerospace Center," in Proc. of SPIE 9647, 2015, no. 9647.

[13] N. Gisin, G. Ribordy, W. Tittel, and H. Zbinden, "Quantum cryptography,” Rev. Mod. Phys., vol. 74, pp. 145$195,2002$.

[14] X.-B. Wang, "Beating the Photon-Number-Splitting Attack in Practical Quantum Cryptography," Phys. Rev. Lett., vol. 94, no. 23, p. 230503, Jun. 2005.

[15] X. Ma, B. Qi, Y. Zhao, and H.-K. Lo, "Practical decoy state for quantum key distribution," Phys. Rev. A, vol. 72, no. 1, p. 012326, Jul. 2005.

[16] V. Scarani, A. Acin, G. Ribordy, and N. Gisin, "Quantum Cryptography Protocols Robust against Photon Number Splitting Attacks for Weak Laser Pulse Implementations,” Phys. Rev. Lett., vol. 92, no. 5, p. 057901, Feb. 2004.

[17] K. Inoue, E. Waks, and Y. Yamamoto, "Differential Phase Shift Quantum Key Distribution," Phys. Rev. Lett., vol. 89, no. 3, p. 037902, Jun. 2002.

[18] D. Stucki, N. Brunner, N. Gisin, V. Scarani, and H. Zbinden, "Fast and simple one-way quantum key distribution," Applied Physics Letters, vol. 87, no. 19, p. 194108, 2005.

[19] J. Lodewyck, M. Bloch, R. Garcıa-Patrón, S. Fossier, E. Karpov, E. Diamanti, T. Debuisschert, N. J. Cerf, R. Tualle-Brouri, S. W. McLaughlin, and P. Grangier, "Quantum key distribution over $25 \mathrm{~km}$ with an all-fiber continuous-variable system," Phys. Rev. A, vol. 76, no. 4, p. 042305, Oct. 2007.

[20] P. Jouguet, S. Kunz-Jacques, A. Leverrier, P. Grangier, and E. Diamanti, "Experimental demonstration of longdistance continuous-variable quantum key distribution," Nature Photonics, vol. 7, no. 5, pp. 378-381, Apr. 2013.

[21] D. Huang, P. Huang, D. Lin, and G. Zeng, "Long-distance continuous-variable quantum key distribution by controlling excess noise," 2019.

[22] V. Scarani, H. Bechmann-Pasquinucci, N. J. Cerf, M. Dušek, N. Lütkenhaus, and M. Peev, "The security of practical quantum key distribution," Reviews of Modern Physics, vol. 81, no. 3, pp. 1301-1350, Sep. 2009.

[23] F. Laudenbach, C. Pacher, C.-H. F. Fung, A. Poppe, M. Peev, B. Schrenk, M. Hentschel, P. Walther, and H. Hübel, "Continuous-Variable Quantum Key Distribution with Gaussian Modulation-The Theory of Practical Implementations," Advanced Quantum Technologies, vol. 1, no. 1, p. 1800011, Jun. 2018.

[24] V. C. Usenko, B. Heim, C. Peuntinger, C. Wittmann, C. Marquardt, G. Leuchs, and R. Filip, "Entanglement of Gaussian states and the applicability to quantum key distribution over fading channels," New Journal of Physics, vol. 14, no. 9, p. 093048, Sep. 2012.

[25] J. Horwath, D. D. Gonzalez, L. M. Navajas, A. L. Souto, V. Semerjyan, G. Raju, J. Grabowsky, C. Garcia, Y. Lai, T. Bähr, C.-C. Chen, F. Haque, A. Grier, H. Harding, M. Hunwardsen, E. Booen, and H. Hemmati, "Test results of error-free bidirectional 10 Gbps link for air-to-ground optical communications," in Free-Space Laser Communication and Atmospheric Propagation XXX, 2018, vol. 10524, pp. 417-430. 